\title{
Tuning Interactions in Reconfigurable Kagome Artificial Spin Ices for Magnonics
}

V.S. Bhat ${ }^{1,2, \text { a) }}$ and D. Grundler ${ }^{1,3}$

1) École Polytechnique Fédérale de Lausanne (EPFL), School of Engineering, Institute of Materials,

Laboratory of Nanoscale Magnetic Materials and Magnonics, 1015 Lausanne, Switzerland

${ }^{2)}$ International Research Centre MagTop, Institute of Physics, Polish Academy of Sciences, PL-02668 Warsaw,

Poland

${ }^{3)}$ École Polytechnique Fédérale de Lausanne, School of Engineering, Institute of Electrical and Micro Engineering, Laboratory of Nanoscale Magnetic Materials and Magnonics, 1015 Lausanne, Switzerland

(Dated: 17 August 2021)

We report broadband spin-wave spectroscopy on kagome artificial spin ices (KASIs) made of large arrays of disconnected $\mathrm{Ni}_{81} \mathrm{Fe}_{19} 400$-nm-long nanobars. We observed spin wave spectra that varied characteristically upon the separation between neighboring nanobars. The dipolar coupling within the KASI is analyzed in terms of a configurationdependent bias field. It varies between about 30 and $40 \mathrm{mT}$ for nearest-neighbor separations of 300 and $60 \mathrm{~nm}$, respectively. Similarly to interconnected KASIs, we reprogramm the spin wave spectra by means of a specific field protocol. The disconnected KASIs are promising for reprogrammable spin wave guiding in underlayers.

Artificial spin ices are created from arrays of nanobars on periodic lattices ${ }^{1,2}$ and have attracted considerable attention from fundamental physics as well as magnonics community ${ }^{3,4}$. Significant progress has been made in understanding quasistatic and magnetodynamics properties at $\mathrm{GHz}$ frequencies in kagome artificial spin ice (KASI) made of arrays of interacting nanobars on kagome lattice ${ }^{2,5-7}$. The artificial spin ices have been proposed to answer not only fundamental questions about geometrical frustration effects $6,8,9$ but also as planar reconfigurable magnonic crystals ${ }^{3,7,10-13}$. One of the crucial questions that still need to be studied in detail is what happens to the spin wave dynamics when one systematically tunes the geometrical frustration via tuning the dipolar interactions among the nanobars. Recently, an interconnected KASI was reprogrammed using a $2 \mathrm{D}$ magnetic field protocol ${ }^{14}$, but no studies have been reported on whether such reprogramming of arrays is possible when exchange interaction among the nanobars is absent.

Here we report our study on the effect of differently engineered dipolar interactions among nanobars arranged on the kagome lattice (Fig. 1). We observed that spin wave spectra varied characteristically as we studied the angular dependence of resonant microwave absorption. We show that a 2D magnetic protocol can be used to program the KASI also in case of disconnected nanobars. The findings are relevant for hybrid magnonic devices for which the artificial spin ice modifies the magnon propagation in a soft magnetic underlayer ${ }^{15}$.

The KASI samples were prepared using ebeam lithography and lift-off techniques. Each KASI [Fig. 1(a)] covered a writefield area of $120 \times 120 \mu \mathrm{m}^{2}$, and was repeated on a $35 \times 5$ array to increase the signal-to-noise ratio for broadband spectroscopy measurements. The separation between neighboring KASI was $10 \mu \mathrm{m}$ along $x$ - and $y$-direction. The nomina length, width, and thickness of a $\mathrm{Ni}_{81} \mathrm{Fe}_{19}$ (Py) nanobar were kept at $400 \mathrm{~nm}, 130 \mathrm{~nm}$, and $25 \mathrm{~nm}$, respectively. The shortest

a) Authors to whom correspondence should be addressed: Vinayak Bhat, vbhat@magtop.ifpan.edu.pl; Dirk Grundler, dirk.grundler@epfl.ch distances between two nanobars in samples named SampleI [Fig. 1(a)] and Sample-II [Fig. 1(b)] were $60 \mathrm{~nm}$ and 300 $\mathrm{nm}$, respectively. Room-temperature broadband spin wave spectroscopy measurements were performed using a coplanar waveguide with 20 microns wide signal line aligned along the $T 1$ nanobars [corresponding to $\phi=0^{\circ}$ in Fig. 1(c)] and vector network analyzer. We recorded the $S$-parameters as a function of frequency $f$ at the constant $\mu_{0} H$ and fixed angle $\phi$. This corresponded to single spectrum $S[H, \phi]$. We then subtracted a spectrum taken at a successive field $H+\delta H$ taken at the same $\phi$ and obtained $\Delta S=S[H+\delta H, \phi]-S[H, \phi]$ providing an enhanced signal-to-noise ratio to identify resonances. The field $\mu_{0} H$ was varied from $-90 \mathrm{mT}$ to $+90 \mathrm{mT}$ in steps of $\mu_{0} \delta H=1 \mathrm{mT}$.

Simulations were performed using the OOMMF code $\mathrm{e}^{7,16}$. The Py parameters used in the simulations were as follows: exchange constant $A=1.3 \times 10^{-11} \mathrm{~J} \mathrm{~m}^{-1}$, saturation magnetization $M_{\mathrm{S}}=820 \times 10^{3} \mathrm{Am}^{-1}$, and dimensionless damping coefficient $\alpha=0.01$. The simulations displayed in Fig. 1(d) to (f) highlight that for $\phi=15^{\circ}$ three types of nanobars $T 1, T 2$, and $T 3$ can be distinguished in that their fundamental resonances of nearly uniform spin precession in (d) and (e) occur at distinctly different eigenfrequencies. Similarly to interconnected KASIs, we reprogramm the spin wave spectra by means of a specific field protocol. These are attributed to different internal fields ${ }^{17}$ due the orientation-dependent demagnetizing effect.

Figure 2 shows detailed spin wave spectra at various fixed angles $\phi$ for Sample-I (top row) and Sample-II (bottom row) as a function of $H$. At $\phi=0^{\circ}$, we observed two prominent branches, A1 and B1, showing spin wave resonance at 13.3 $\mathrm{GHz}$ and $10.6 \mathrm{GHz}$, respectively, at $\mu_{0} H=-90 \mathrm{mT}$ in Sample-I. The spin wave frequencies of branches A1 and B1 decrease as we increase $H$. The two branches cross one another at $\mu_{0} H=+12 \mathrm{mT}$. At $\mu_{0} H=44 \mathrm{mT}$, we see jumps in the branches (switching fields of modes) and at $48 \mathrm{mT}$ all the nanobars have undergone the switching process. At $\phi=5^{\circ}$, another spin wave branch appears in that the single branch B1 splits into two. As $\phi$ is increased further to $15^{\circ}$, mode B1 


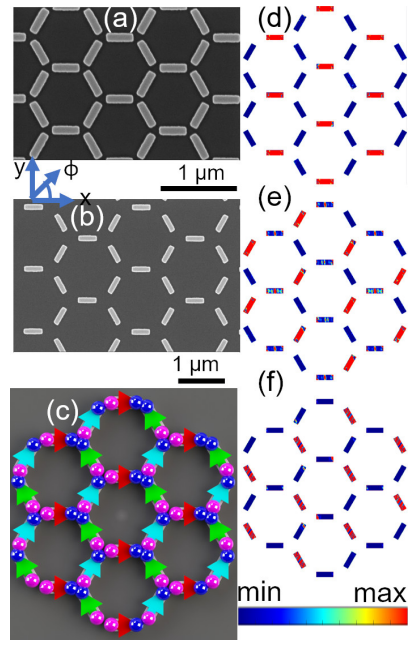

FIG. 1. Microscopy images of inner sections of (a) Sample-I and (b) Sample-II. Bright (dark) regions correspond to $\mathrm{Ni}_{81} \mathrm{Fe}_{19}$ (Si). (c) Cartoon representation of magnetization vectors $\mathbf{M}$ (arrows) in the KASI sample when the $T 1$ (red) and $T 2$ (cyan) nanobars have undergone switching while T3 (green) nanobars are still aligned with the initial saturating field. The blue and magenta balls represent charges $+q$ and $-q$, respectively. Spin precessional power maps for SampleII at $\mu_{0} H=-90 \mathrm{mT}$ applied at $\phi=15^{\circ}$ illustrate prominent power absorption in (d) $T 1$ (at $f=13.2 \mathrm{GHz}$ ), (e) $T 2$ (at $f=11.6 \mathrm{GHz}$ ), and (f) $T 3$ (at $f=9.5 \mathrm{GHz}$ ) nanobars. The red and blue color represents maximum $\left[200(\mathrm{~A} / \mathrm{m})^{2}\right]$ and minimum $\left[0(\mathrm{~A} / \mathrm{m})^{2}\right]$ power $\left(\mathrm{M}_{\mathrm{z}}{ }^{2}\right)$ absorption, respectively.

splits more clearly into the two modes B 1 and $\mathrm{C} 1$. The simulations performed for $15^{\circ}$ and $-90 \mathrm{mT}$ [Fig. 1(d) to (f)] allow us to attribute the branches $\mathrm{A} 1, \mathrm{~B} 1$, and $\mathrm{C} 1$, to the three differently oriented nanobars $T 1, T 2$, and $T 3$, respectively. The mode $\mathrm{B} 1$ moves to the higher frequency side as the in-plane angle is increased, whereas resonance frequencies of modes A1 and C1 decrease.

For Sample-II, we again observed two branches with resonance frequencies similar to Sample-I. This implies that the resonance frequencies for the saturated regime depend upon the length, width, and thickness of a nanobar. The separation between the nanobars does not play a major role for frequencies at large $H$ indicating that the dipolar coupling and stray fields do not contribute significantly to the internal fields at high fields ${ }^{17}$. The spin wave frequencies of branches A2 and B2 decrease as we sweep to positive $H$. The two branches cross one another at $\mu_{0} H=+4 \mathrm{mT}$. This value is smaller compared to Sample-I. At about $44 \mathrm{mT}$, we observe jumps in the spin wave branches representing switching of nanobars into the applied field direction. At $48 \mathrm{mT}$ all the nanobars have undergone the switching process, similar to Sample-I. At $\phi=$ $5^{\circ}$, a separate spin wave branch appears. As $\phi$ is increased further, the mode B2 splits into two modes B2 and C2, allowing for the allocation to $T 2$ and $T 3$ nanobars, respectively. The mode B2 moves towards higher frequency, whereas resonance frequencies of modes $\mathrm{A} 2$ and $\mathrm{C} 2$ get lower as the in-plane angle increases and reach their extreme values at $30^{\circ}$.

The symbols in Fig. 2 highlight that the branches A (red symbols), B (green symbols), and C (cyan symbols) arise from the power absorption due to the fundamental modes in nanobars of types $T 1, T 2$, and $T 3$, respectively. Additional branches corresponding to absorption due to higher-order standing spin waves in $T 1$ (blue symbols) and $T 2$ (yellow symbols) nanobars were also seen in simulations and experiments. They reside at lower frequencies compared to branches $\mathrm{A}$ and $\mathrm{B}$, respectively, indicating confinement along the long axis, i.e., confined backward volume magnetostatic spin waves.

We observe distinct features in the switching regime of Sample-I when $\phi>5^{\circ}$ : 1) The switching field of spin wave modes A1 and B1 remains almost identical to the one for $\phi=$ $0^{\circ}$, whereas the switching field of $\mathrm{C} 1$ greatly varies with angle. 2) The previous study ${ }^{18}$ on KASI made out of interconnected nanobars has reported the appearance of a single branch representing switched $T 1$ and $T 2$ segments that showed a change in slope as the branch corresponding to switched $T 3$ segments emerged. For disconnected nanobars studied here observations are different: i) two separate branches corresponding to switched $T 1$ and $T 2$ segments are resolved. ii) The slopes $d f / d H$ of branches $\mathrm{A} 1$ and $\mathrm{B} 1$ attributed to $T 1$ and $T 2$ segments, respectively, after switching get similar as $\phi$ approaches $30^{\circ}$. iii) The slopes $d f / d H$ of branches $\mathrm{A} 1$ and $\mathrm{B} 1$ undergo changes as the branch $\mathrm{C} 1$ starts to emerge. iv) After the switching of $T 3$ nanobars, the branch corresponding to $T 2$ shifts to a lower frequency, whereas the branch corresponding to $T 1$ shifts to a higher frequency. In Fig. 3(a) [(b)] we show the values $d f / d H$ of branches A1 and A2 of SampleI (Sample-II) as extracted from the measured resonance frequencies $f$ at $\mu_{0} H>50 \mathrm{mT}$ [c.f. Fig. 3(c)], i.e., after the switching of $T 1$ and $T 2$ nanobars, for different $\phi$. For SampleI and $\phi=10,15$, and $20^{\circ}$ we observe local maxima in $d f / d H$ near 64,71 , and $79 \mathrm{mT}$, respectively. The upper curve $f(H)$ (symbols) for $\phi=15^{\circ}$ in Fig. 3(c) substantiates that the local maximum in $d f / d H$ identifies the field regime where branch A of Sample-I gains a frequency offset to higher frequencies with increasing $H$. We attribute this field regime to the fields for which $T 3$ nanobars switch. The local maxima in $d f / d H$ are not clearly developed for Sample-II in Fig. 3(b), consistent with the nearly vanishing frequency offset of branch A displayed by the lower curve (symbols) in Fig. 3(c). We attribute the latter observations to the relatively large separation between nanomagnets which reduces the dipolar interaction by stray fields. This will be further analyzed in the following. In the switching regime of $T 3$ nanobars, we see a pronounced characteristic difference between Sample-I and Sample-II [compare the white dashed arrows in Fig. 2(d) and (k)] as the applied field lifts the degeneracy for $\phi>5^{\circ}$ : the two branches A and B change their slopes $d f / d H$ differently when branch $\mathrm{C}$ appears [Figs. 3(a) and (b)]. We consider these changes in $d f / d H$ to be a direct function of the separation between the 


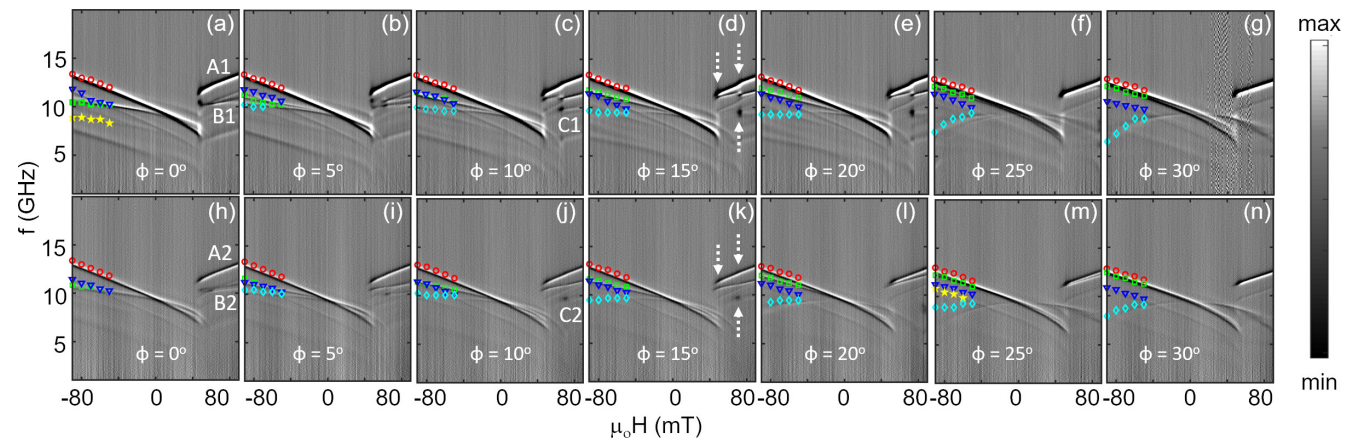

FIG. 2. Spin wave absorption spectra $\Delta S$ for (a-g) Sample-I (top row) and (h-n) Sample-II (bottom-row), for various in-plane angles $\phi$. The dashed vertical arrows in (d) and (k) define regions in which Type $T 1$ and $T 2$ nanobars are switched but $T 3$ nanobars remain unswitched. The min and max in the grey scale map corresponds to -0.0001 and 0.0001 in arbitrary units. The symbols correspond to resonance frequency values extracted from simulations.

nanobars. To explore this consideration in further detail, we make use of the charge model ${ }^{5,8,19}$. We assume that each Py nanobar is a dumbbell with charges $+q=M \cdot t \cdot w \cdot l / l$ and $-q$ at its opposing ends, where $M$ represents the magnetization of a given Py nanobar. Each vertex in a KASI (excluding the outermost rim) possesses a coordination number of 3 , and the total charge at a given vertex is defined as $Q=\sum_{i}^{3} q_{i}$. If one considers the switching regime where the nanobars $T 1$ and $T 2$ undergo switching but not $T 3$, one observes that the total charge remains the same as in the completely un-switched reference configuration. However, the $-q$ charge of a $T 2$ nanobar is in close proximity with the $+q$ charge of the $T 1$ and $T 3$ nanobars. Repulsion (and attraction) of these charges displays the dipolar interaction between nanobars and potentially modifies resonance frequencies at moderate applied fields. The situation reverses when the nanobars of type $T 3$ reverse because the $-q$ charge of $T 2$ nanobar is in close proximity with the $-q$ charge of the $T 3$.

We now discuss possible reasons for different $d f / d H$ in Fig. 3(a) and (b) focusing on $\phi=15^{\circ}$. We assume that the local magnetic configuration of neighboring nanobars induces a bias magnetic field $H_{\mathrm{B}}$, which indicates their effective dipolar interactions. To estimate this field, we consider the Kitte equation for an individual magnetic ellipsoid in which we introduce $H_{\mathrm{B}}$ as an additional magnetic field: ${ }^{3,17}$

$f=\frac{\gamma \mu_{0}}{2 \pi} \sqrt{\left[\left(H+H_{\mathrm{B}}\right)+\left(N_{z}-N_{x}\right) M\right]\left[\left(H+H_{\mathrm{B}}\right)+\left(N_{y}-N_{x}\right) M\right]}$

where $\gamma$ is the gyromagnetic ratio and $\gamma / 2 \pi=28 \mathrm{GHz} / \mathrm{T}$ For the nanobars we estimate relevant demagnetization factors $N_{x}$ (along the long axis), $N_{y}$ (across the width), and $N_{z}$ (in out-of-plane direction) using Ref. ${ }^{20}$. Note that $H_{\mathrm{B}}$ is not an absolute measure of the interaction field as Eq. (1) assumes the alignment of both $\mathbf{H}$ and $\mathbf{M}$ with the nanobar's long axis. This is not strictly realized for $\phi=15^{\circ}$, but the field component $H \times \sin 15^{\circ}$ is considerably smaller than the anisotropy field $H_{\text {ani }}$ (see below) and allows for a small misalignment angle only. Under these conditions, $H_{\mathrm{B}}$ allows us to relatively compare the effect of the different separations of nominally identical nanobars. $H_{\mathrm{B}}=0$ would correspond to an isolated nanobar without interacting neighbors whose eigenfrequency is described by the unmodified Kittel equation. Assuming $N_{x}+N_{y}+N_{z}=1$ we get values $N_{x}=0.05457$, $N_{y}=0.1758$, and $N_{z}=0.7695$ for the 400-nm-long nanobars with magnetization $\mu_{0} M=1.03 \mathrm{~T}$. These values suggest $\mu_{0} H_{\text {ani }}=\left(N_{y}-N_{x}\right) \mu_{0} M \approx 0.12 T>\mu_{0} H$. We fit Eq. (1) to different segments of branches A of Sample-I and Sample-II in Fig. 3(c) (colored lines). The bias magnetic fields $\mu_{0} H_{\mathrm{B}}$ evaluated for $T 1$ nanobars after (before) the switching of $T 3$ nanobars in Sample-I and Sample-II amount to $39.8 \pm 0.07$ $\mathrm{mT}(33.1 \pm 0.45 \mathrm{mT})$ and $30.7 \pm 0.12 \mathrm{mT}(28.5 \pm 0.21$ $\mathrm{mT})$, respectively. The value $\mu_{0} H_{\mathrm{B}}$ is higher for the closely spaced nanobars in Sample-I. The differences between the internal fields before and after switching of $T 3$ nanobars are 6.7 and $2.2 \mathrm{mT}$ for Sample-I and Sample-II, respectively. The large (small) difference of 6.7 (2.2) mT extracted for SampleI (Sample-II) substantiates why local maxima $d f / d H$ are well (not) resolved in Fig. 3(a) [(b)].

Recently, interconnected KASI were reconfigured using a 2D magnetic field protocol ${ }^{14}$. It is instructive to see whether disconnected KASI ${ }^{21-23}$ can be reconfigured as well. We demonstrate how to reconfigure the kagome ASI between configurations $\mathrm{X}$ and $\mathrm{Y}$ of bow-tie subgroups ${ }^{14}$ which are sketched on top of Fig. 4. We first sweep the field from $\mu_{0} H=90 \mathrm{mT}$ at $\phi$ $=15^{\circ}$ to $-70 \mathrm{mT}$ and then to $0 \mathrm{mT}$ at the same angle $\phi$. Up to $0 \mathrm{mT}$ the branches are monotonic, indicating that $\mathrm{X}$ is stable at the remnant configuration. At $0 \mathrm{mT}$, we change the angle setting to $\phi=-15^{\circ}$. Subsequently, we increase the field from 0 to $90 \mathrm{mT}$ along $\phi=-15^{\circ}$. At $\phi=-15^{\circ}$, the vectors $\mathbf{M}$ of $T 1$ 


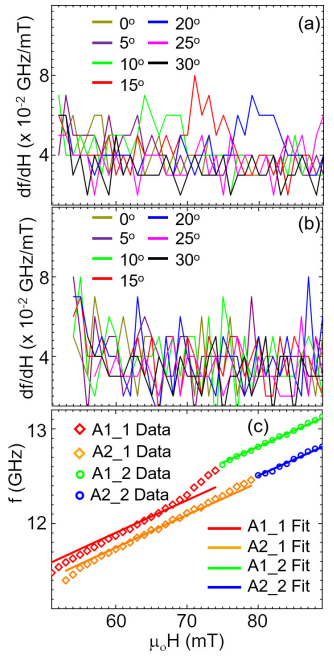

FIG. 3. Slope $d f / d H$ extracted from branches A measured at different angles $\phi$ for (a) Sample-I and (b) Sample-II. (c) Field dependencies of branches A1 1 (A2 1) and A1 2 (A2 2) for Sample-I (Sample-II) before (_1) and after (_2) the switching of T3 nanobars. The angle $\phi$ was $15^{\circ}$. Open symbols represent the resonance frequency values at $\phi=15^{\circ}$ and the solid lines represent fits using Eq. (1) in the different segments of branches A

(red) and $T 2$ (blue) nanobars are opposed to $\mathbf{H}$, whereas $\mathbf{M}$ of $T 3$ (green) nanobars of bow-tie-subgroup configuration $\mathrm{X}$ are aligned with $\mathbf{H}$. Therefore, only the $T 3$ nanobars lead to a branch with $d f / d H>0$, while the other two types of nanobars induce branches with $d f / d H<0$. At about $+45 \mathrm{mT}$ in Fig. 4(a) and (b) we see that a high-frequency branch A1 corresponding to switched $T 1$ nanobars (red) has emerged. Due to the smaller misalignment angle with $\mathbf{H}$ their resonance is at a higher frequency than the branch of the aligned $T 3$ nanobars. These latter nanobars do not change their magnetization direction for increasing $H$. We call this emerging configuration in the bow-tie subgroup Y. In the intermediate field regime between 45 and $75 \mathrm{mT}$ (configuration $\mathrm{Y}$ ) only the central $T 1$ nanobar out of five nanobars of the bow-tie subgroup is oriented differently compared to configuration X. But, the dynamic response of the KASI is completely different. This is shown by the dynamic simulations performed for configuration X with $-50 \mathrm{mT}$ at $\phi=15^{\circ}$ and $\mathrm{Y}$ with $50 \mathrm{mT}$ at $\phi=-15^{\circ}$ in Fig. 4(c) to (h). We observe that when $T 1$ nanobars are aligned with the field direction, they resonate at $11.81 \mathrm{GHz}$ [Figs. 4(c) and (f)]. This confirms that the field protocol induces switching of $T 1$ nanobar in configuration Y compared to X. Simulated power maps in Figs. 4(d) and (e) for configuration $\mathrm{X}$ show that $T 2$ and $T 3$ nanobars prominently resonate at $f=11.23 \mathrm{GHz}$ and $f=8.3 \mathrm{GHz}$, respectively, indicating

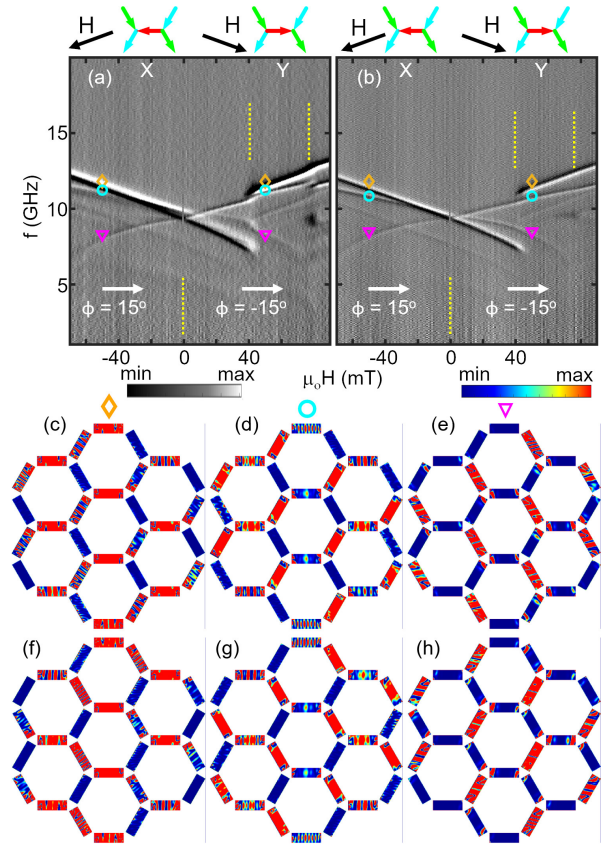

FIG. 4. Gray scale spectra $\Delta S$ of (a) Sample-I and (b) Sample-II for a minor loop with $90 \mathrm{mT} \rightarrow-70 \mathrm{mT} \rightarrow 0 \mathrm{mT}$ at $\phi=15^{\circ}$ and then $0 \rightarrow+90 \mathrm{mT}$ at $\phi=-15^{\circ}$. The solid white arrows indicate field sweep directions. The dashed yellow lines highlight regimes attributed to particular nanobar configurations shown on the top. The black arrows indicate field directions with respect to the displayed bow-tie subgroup. The min and max in the grey scale map corresponds to -0.0001 and 0.0001 in arbitrary units. Spin precessional maps for configuration $\mathrm{X}$ at $\mu_{0} H=-50 \mathrm{mT}$ and $\phi=15^{\circ}$ at $f=$ (c) $11.81 \mathrm{GHz}$, (d) $11.23 \mathrm{GHz}$, and (e) $8.3 \mathrm{GHz}$, and configuration $\mathrm{Y}$ at $\mu_{0} H=50 \mathrm{mT}$ and $\phi=-15^{\circ}$ at $f=$ (f) $11.81 \mathrm{GHz},(\mathrm{g}) 11.23 \mathrm{GHz}$, and (h) $8.3 \mathrm{GHz}$ for Sample-I. The red and blue color represents maximum $\left[200(\mathrm{~A} / \mathrm{m})^{2}\right]$ and minimum $\left[0(\mathrm{~A} / \mathrm{m})^{2}\right]$ power $\left(\mathrm{M}_{\mathrm{z}}{ }^{2}\right)$ absorption at $\mu_{0} H=50 \mathrm{mT}$ and $\phi=-15^{\circ}$, respectively.

that $T 2$ nanobars are switched but $T 3$ nanobars are not. For configuration $\mathrm{Y}$ in Figs. 4(g) and $(\mathrm{h})$ the resonance frequency of $T 3$ nanobar is however higher as compared to $T 2$ nanobars, i.e., the frequency hierarchy is reversed compared to X. This is in line with the experimental findings. The presented twodimensional field protocol allows one to reconfigure KASIs systematically and utilize their distinctly different spin wave spectra and modified stray field distribution for reconfigurable magnonic crystal applications ${ }^{15}$.

To summarize, we have studied KASI with varying separations between the nanobars and thereby tuned the dipolar interactions. We observe angle-dependent spin wave modes 
that show a characteristic dependence on the dipolar interaction. We reconfigured kagome ASI consisting of disconnected nanobars governed by dipolar interaction only using a 2D magnetic field protocol. Our findings pave the way for the usage of KASI as a reconfigurable magnonic crystals and waveguiding.

\section{ACKNOWLEDGMENTS}

The research was supported by the Swiss National Science Foundation via Grant No. 163016. V.S. Bhat acknowledges support from the foundation for Polish Science through the

IRA Programme financed by EU within SG OP Programme.

The data that support this study will be made available on the Zenodo repository during the publication of the manuscript.

${ }^{1}$ R. Wang, C. Nisoli, R. Freitas, J. Li, W. McConville, B. Cooley, M. Lund, N. Samarth, C. Leighton, V. Crespi, et al., Nature 439, 303 (2006).

${ }^{2}$ Y. Qi, T. Brintlinger, and J. Cumings, Physical Review B 77, 094418 (2008)

${ }^{3}$ X. Zhou, G.-L. Chua, N. Singh, and A. O. Adeyeye, Advanced Functional Materials 26, 1437 (2016).

${ }^{4}$ M. Krawczyk and D. Grundler, J. Phys.: Condens. Matter 26, 123202 (2014)

${ }^{5}$ P. Mellado, O. Petrova, Y. Shen, and O. Tchernyshyov, Phys. Rev. Lett. 105, 187206 (2010).
${ }^{6}$ S. Ladak, D. Read, G. Perkins, L. Cohen, and W. Branford, Nature Physics 6, 359 (2010).

${ }^{7}$ V. Bhat, F. Heimbach, I. Stasinopoulos, and D. Grundler, Physical Review B 93, 140401 (2016).

${ }^{8}$ E. Mengotti, L. J. Heyderman, A. F. Rodríguez, F. Nolting, R. V. Hügli, and H.-B. Braun, Nat. Phys. 7, 68 (2011).

${ }^{9}$ S. Gliga, A. Kákay, R. Hertel, and O. G. Heinonen, Phys. Rev. Lett. 110, 117205 (2013)

${ }^{10}$ D. M. Arroo, J. C. Gartside, and W. R. Branford, Physical Review B 100, 214425 (2019)

${ }^{11}$ S. Gliga, E. Iacocca, and O. G. Heinonen, APL Materials 8, 040911 (2020). ${ }^{12}$ L. Heyderman and R. Stamps, J. Phys.: Condens. Matter 25, 363201 (2013).

${ }^{13}$ M. Jungfleisch, W. Zhang, E. Iacocca, J. Sklenar, J. Ding, W. Jiang, S. Zhang, J. Pearson, V. Novosad, J. Ketterson, et al., Physical Review B 93, 100401 (2016).

${ }^{14}$ V. Bhat, S. Watanabe, K. Baumgaertl, A. Kleibert, M. Schoen, C. Vaz, and D. Grundler, Physical Review Letters 125, 117208 (2020).

${ }^{15}$ E. Iacocca, S. Gliga, and O. G. Heinonen, Physical Review Applied 13, 044047 (2020).

${ }^{16} \mathrm{M}$. Donahue and D. Porter, Interagency Report NISTIR 6376 (1999)

${ }^{17}$ A. G. Gurevich and G. A. Melkov, Magnetization oscillations and waves (CRC Press, 1996.)

${ }^{18}$ V. S. Bhat, F. Heimbach, I. Stasinopoulos, and D. Grundler, Physical Review B 96, 014426 (2017)

${ }^{19}$ C. Castelnovo, R. Moessner, and S. L. Sondhi, Nature 451, 42 (2008)

${ }^{20}$ A. Aharoni, Journal of Applied Physics 83, 3432 (1998).

${ }^{21}$ A. Talapatra, N. Singh, and A. Adeyeye, Physical Review Applied 13, 014034 (2020).

${ }^{22}$ W. Bang, J. Sturm, R. Silvani, M. T. Kaffash, A. Hoffmann, J. B. Ketterson, F. Montoncello, and M. B. Jungfleisch, Physical Review Applied 14, 014079 (2020)

${ }^{23}$ T. Dion, D. Arroo, K. Yamanoi, T. Kimura, J. Gartside, L. Cohen, H. Kurebayashi, and W. Branford, Physical Review B 100, 054433 (2019). 

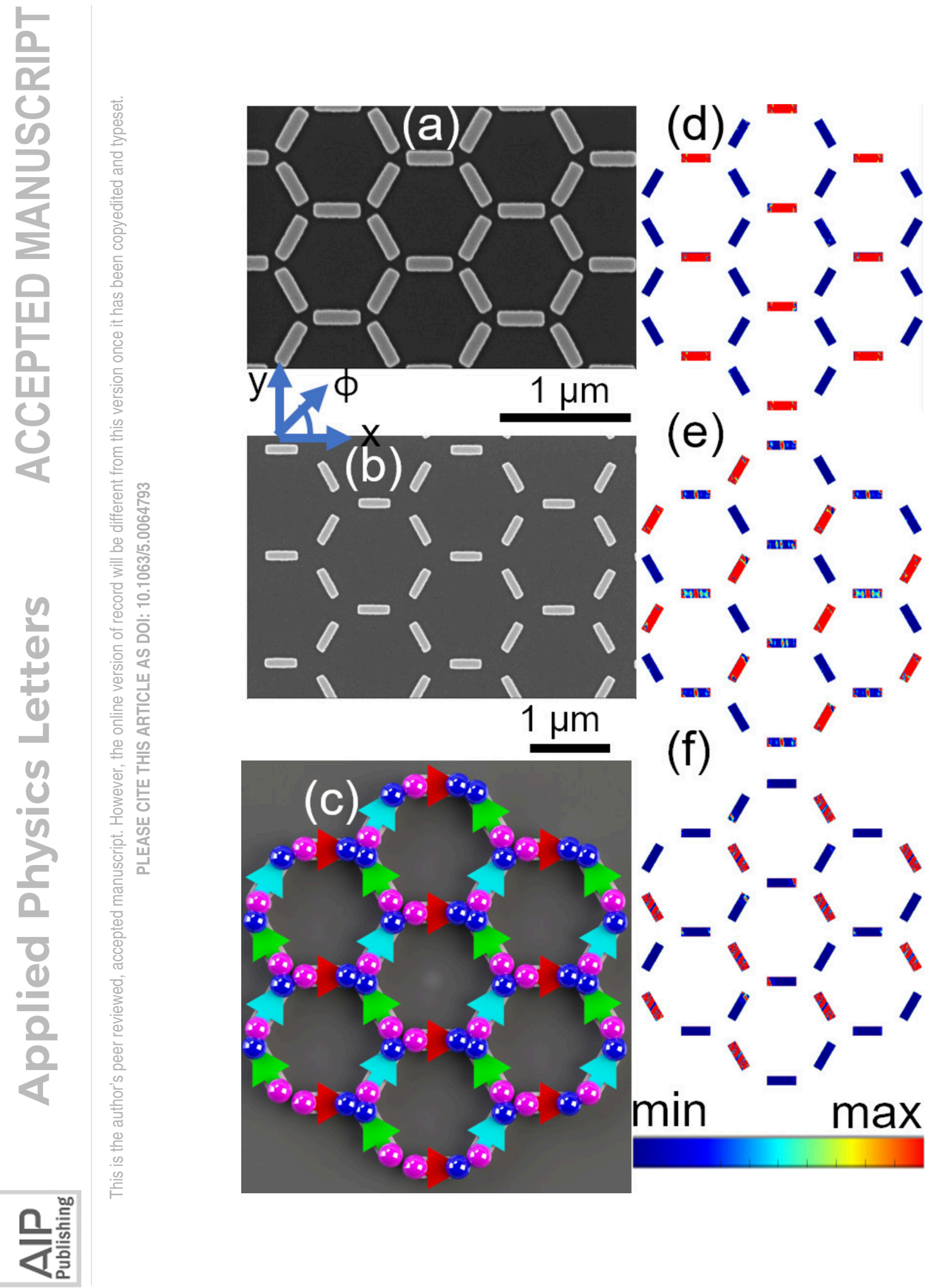


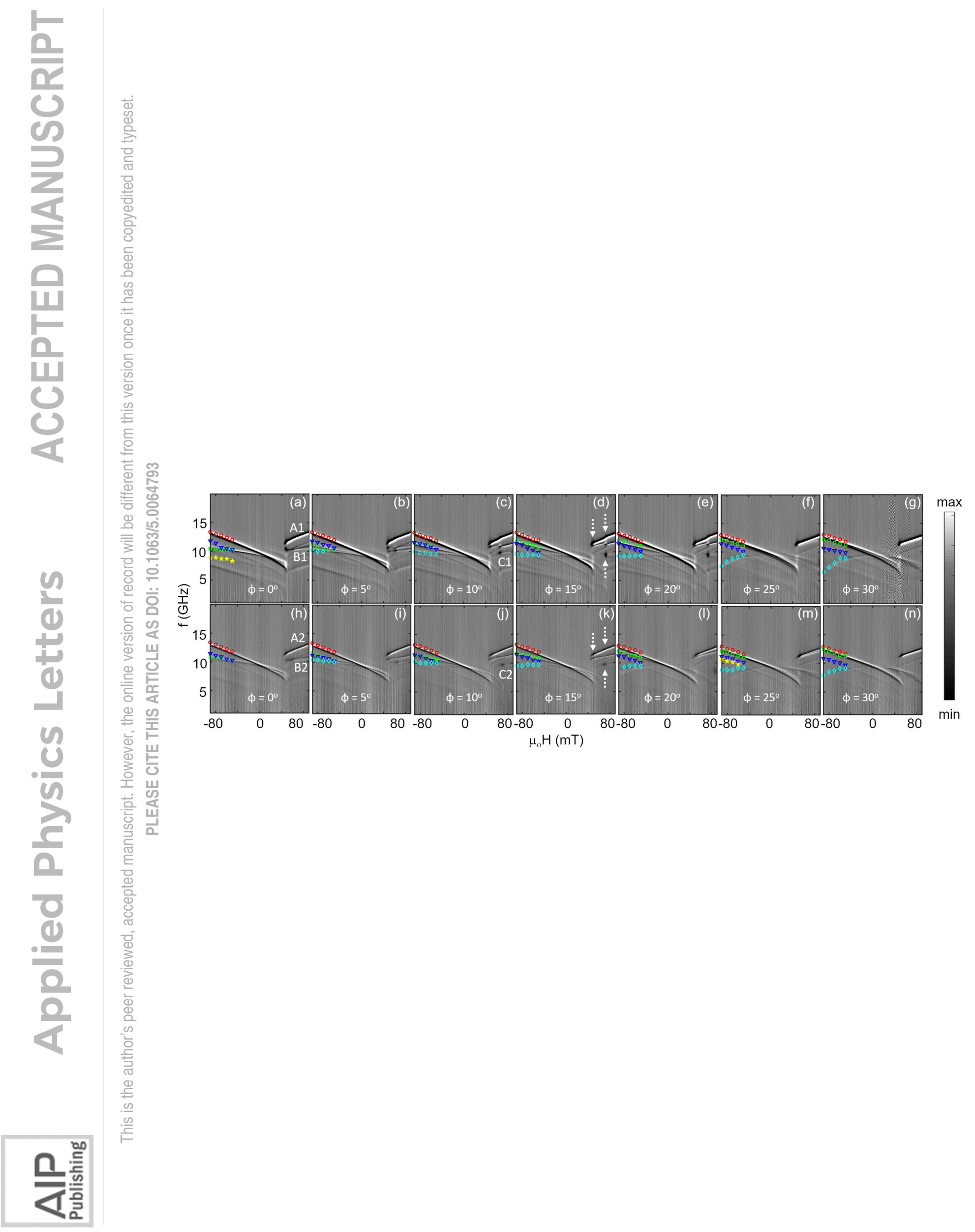




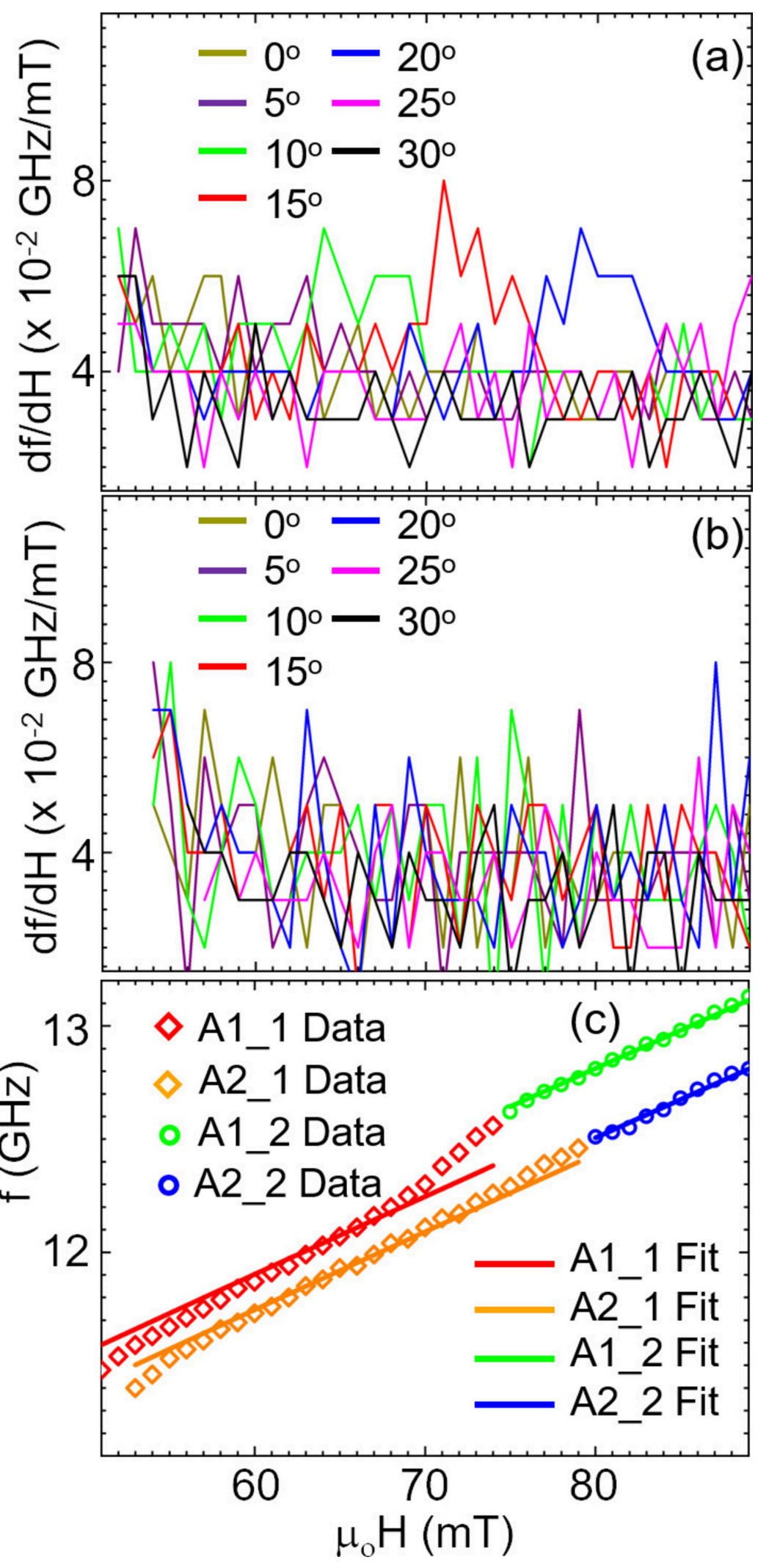




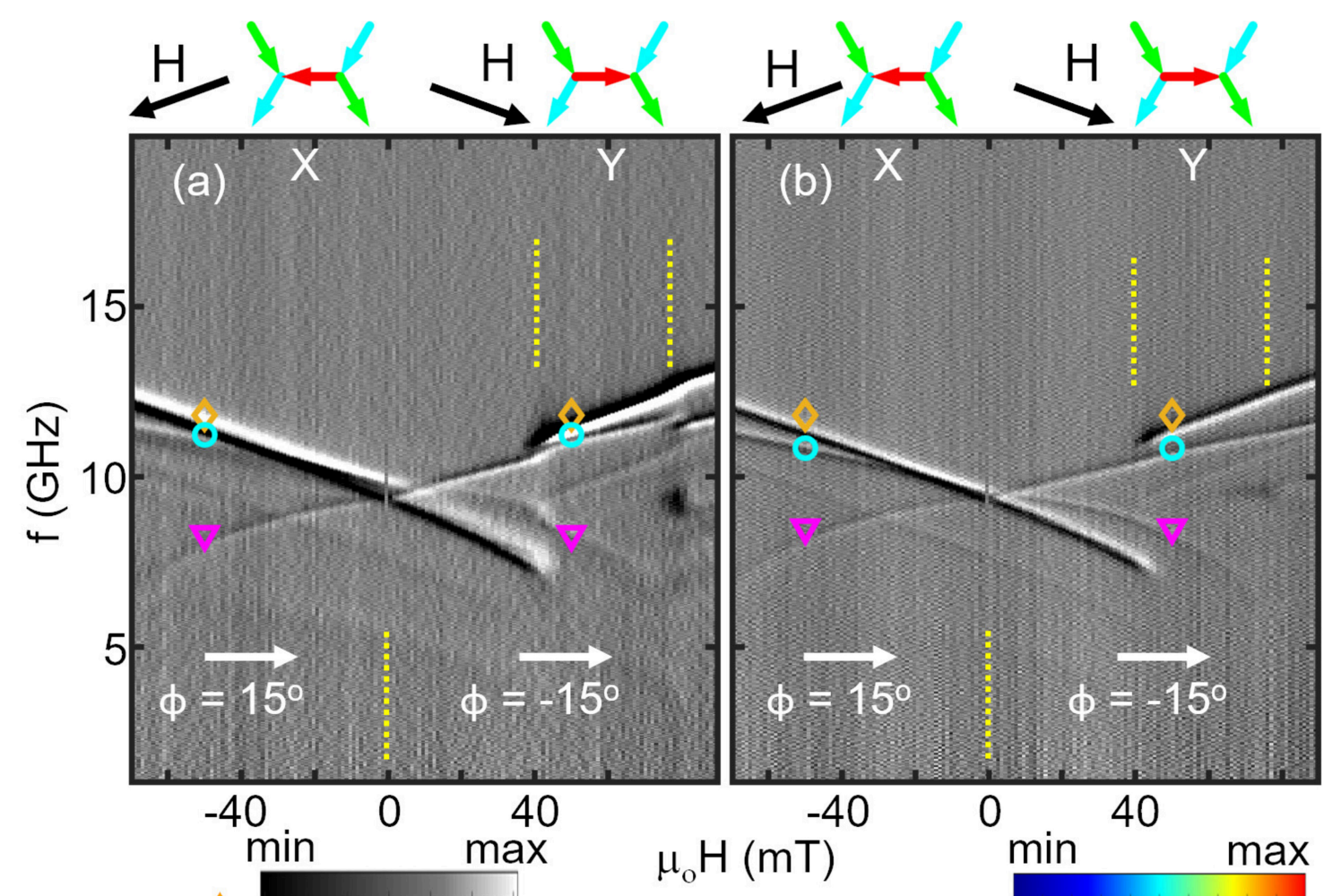

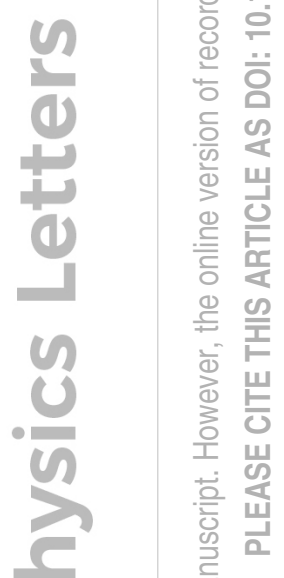
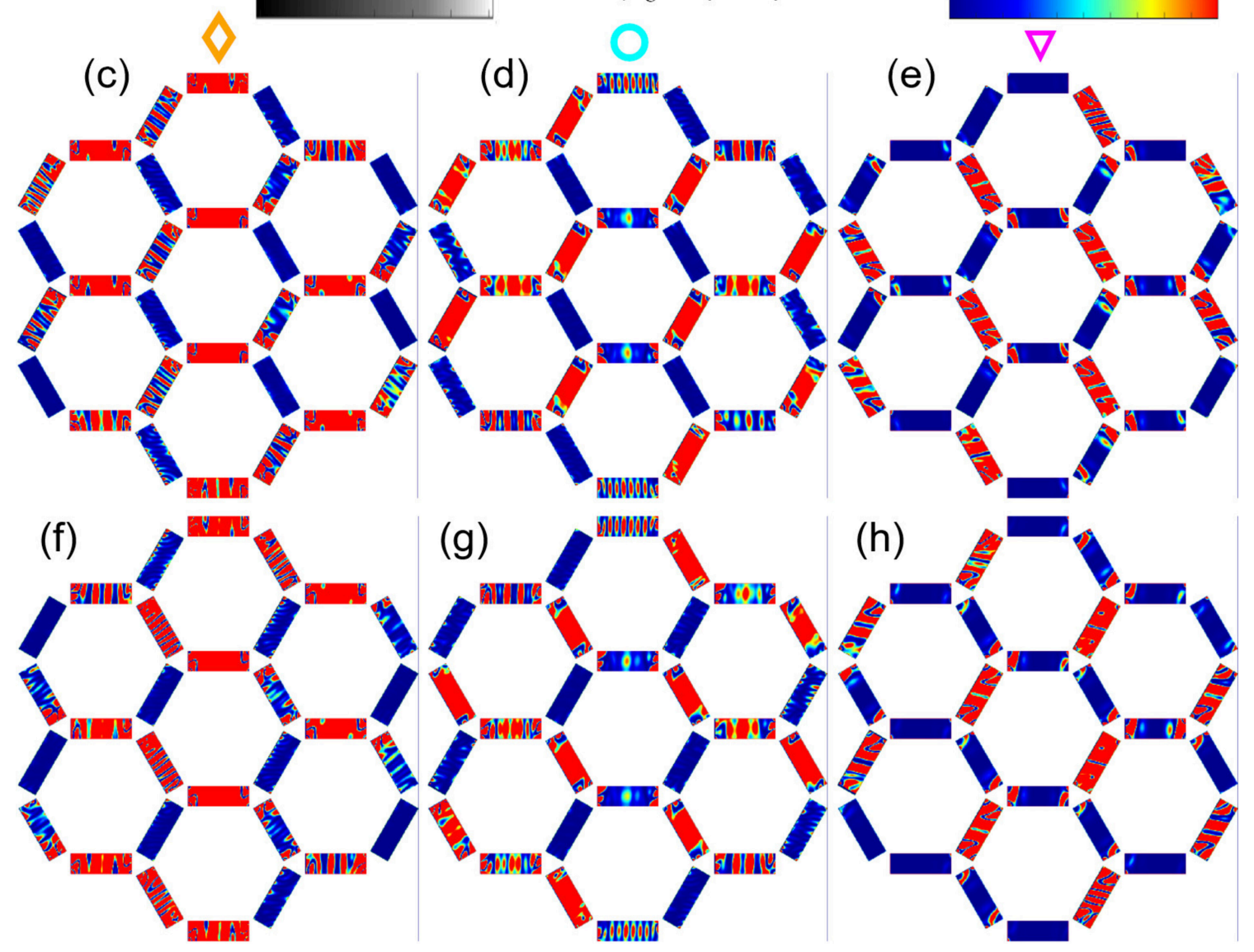

(g)

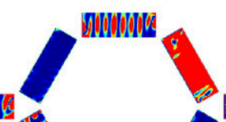

(h) 